\title{
Maternal serum zinc levels and urinary tract infections among pregnant women at a tertiary hospital in Lusaka, Zambia: a cross-sectional study
}

\author{
John Musaba ${ }^{1}$, Moses Mukosha ${ }^{2 *}$, Lubinda Mukololo ${ }^{1}$, Bellington Vwalika ${ }^{3}$
}

\begin{abstract}
${ }^{1}$ Department of Physiological Sciences, School of Medicine, ${ }^{2}$ Department of Pharmacy, School of Health Sciences, ${ }^{3}$ Department of Obstetrics and Gynaecology, The University of Zambia, Lusaka, Zambia
\end{abstract}

Received: 07 July 2021

Revised: 05 August 2021

Accepted: 06 August 2021

*Correspondence:

Moses Mukosha,

Email: Mukoshamoses@yahoo.com

Copyright: ( ) the author(s), publisher and licensee Medip Academy. This is an open-access article distributed under the terms of the Creative Commons Attribution Non-Commercial License, which permits unrestricted non-commercial use, distribution, and reproduction in any medium, provided the original work is properly cited.

\begin{abstract}
Background: Micronutrient deficiency is one of the leading public health problems, particularly in lower-middleincome countries. The link between serum zinc ( $\mathrm{Zn}$ ) levels and urinary tract infections (UTIs) among pregnant women is still poorly understood. We investigated the association between maternal serum Zn levels and UTIs among pregnant women at women and newborn hospital in Lusaka, Zambia.

Methods: We conducted an analytical cross-sectional study among 228 pregnant women aged 18 to 45 years between 1 September and 30 November 2019. Serum Zn levels were measured using atomic absorption spectrophotometry (AAS), whereas UTIs were determined based on the local hospital guidelines. We used the logistic regression model to determine the association between $\mathrm{Zn}$ levels and UTIs. The analysis was done at a significance level of 5\% and $95 \%$ confidence interval.

Results: Of the total participants, 118/228 (51.75\%) tested positive for UTIs from urine culture. The mean serum Zn levels in the group diagnosed with UTIs were $53.95 \mathrm{ug} / \mathrm{dl}(\mathrm{SD}=35.10)$, while in those who had no UTIs, it was 50.05 $\mathrm{ug} / \mathrm{dl}(\mathrm{SD}=35.10)$. In a multivariable logistic regression analysis, we found no evidence of an association between serum Zn levels and UTIs $(\mathrm{p}=0.435)$.

Conclusions: From the study findings, there was no association between serum Zn levels and UTIs. This result suggests that $\mathrm{Zn}$ may not have any role in urinary tract infection. Nevertheless, more extensive studies are needed to confirm our findings.
\end{abstract}

Keywords: Zinc, Urinary tract infections, Pregnancy, Women and newborn hospital, Zambia

\section{INTRODUCTION}

$\mathrm{Zn}$ is an element involved in protein, DNA and RNA synthesis and plays a role in stabilising cell membranes and the skeletal system. ${ }^{1}$ It is a co-factor for more than 200 enzymes that include metalloenzymes, oxidoreductases, transferases and phosphatases and it is thought to play a pivotal role in immune system function and antioxidant status. ${ }^{1-3} \mathrm{Zn}$ plays a role in intoxicating pathogens such as
E. coli, preventing infections caused by them. ${ }^{4}$ Its deficiency exposes pregnant women to various infections that are due to cell and antibody-mediated immune dysfunction. This is because $\mathrm{Zn}$ helps produce immunoglobulin $\mathrm{G}$ and B-lymphocytes that help boost the immune system and improve humoral and cellular immune responses. ${ }^{5,6} \mathrm{Zn}$ is central to all immune system cells' functioning and serves as a co-factor of thymulin, a hormone involved in immature T-cell differentiation. ${ }^{\text {? }}$ 
$\mathrm{Zn}$ deficiency is common among pregnant women, with an estimated prevalence of $82 \%$ due to an increased micronutrient requirement during pregnancy. ${ }^{8} \mathrm{Zn}$ deficiency increases the risk of UTIs and other infections in pregnancy because it plays a central role in the immune system. ${ }^{8,9}$ It can reduce the adaptive immune response in that it causes thymic atrophy and subsequently, T-cell lymphopenia and the production of premature B-cells, which reduces antibody production. ${ }^{7} \mathrm{Zn}$ deficiency reduces the effect of some innate immune system cells, the first line of defence against pathogens. ${ }^{7}$ Few studies have focused on the role of $\mathrm{Zn}$ in UTIs, specifically in pregnant women.

UTIs are inflammations caused by microbial colonisation of the lower and upper urinary tract. ${ }^{10}$ They are the most common type of infection during pregnancy, with an estimated prevalence of $20 \%$ worldwide and are thought to affect mostly Asia and Sub-Saharan Africa with a prevalence of up to $74 \% .{ }^{11-13}$ In Zambia, the prevalence of UTIs among pregnant women in the study setting in 2019 was $16.5 \% .{ }^{14}$ Pregnant women are more susceptible to developing UTIs, mainly due to physiological changes during pregnancy, especially to the bladder and ureter. These changes are mainly caused by gravid pressure by the uterus on the urinary tract and progesterone's overproduction, which causes ureteral muscle relaxation and consequently, accumulation of urine in the bladder. ${ }^{15}$ These lead to urine flow stasis, which raises the chances of infection. ${ }^{16-18}$

Some studies across the globe reported contradicting results about $\mathrm{Zn}$ and its relationship with UTI. For example, some showed that high $\mathrm{Zn}$ concentrations inhibited the growth of some specific UTIs-causing agents, whereas others showed that high $\mathrm{Zn}$ increased persistence of multi-resistant $E$. coli. ${ }^{7,16,17,19-21}$ Few studies have attempted to explore the role of zinc in the occurrence of UTIs in Sub-Saharan Africa (SSA) and Zambia, particularly among pregnant women.

Even though UTIs are responsible for increased maternal morbidity and mortality in SSA, the determinants are still poorly documented, mainly in pregnant women. Given the differences in ethno-geographic risk factors and the high rate of maternal morbidity in SSA and the uncertainty of whether zinc affects the rate of UTIs, it is critical to understand how the two conditions interact.

Therefore, this study determined the association between serum Zn levels and UTIs among pregnant women presenting to women and newborn hospital in Lusaka.

\section{METHODS}

\section{Study design, setting and population}

This study was an analytical cross-sectional conducted at women and newborn hospital of the university teaching hospitals (UTHs), Lusaka, Zambia. The hospital was the largest referral hospital in Zambia, attending to about 28800 pregnant women per year, with a record of about 18000 births per year. ${ }^{22}$ We enrolled pregnant women of childbearing age (15-49 years of age) attending antenatal clinic between 1 September and 30 November 2019 at the hospital who were willing to participate by signing a written consent.

\section{Enrolment procedure and sample collection}

Systematic random sampling was used to enrol pregnant women with a sampling interval of 2 (every 2 nd pregnant woman, 398/117th) for every pregnant woman who met the eligibility criteria. A validated screening questionnaire and a signed consent form were used to determine participation. Women were considered eligible if they were pregnant and Zambian, and were 15 years of age and above and were not participating in any conflicting protocol or were non-diabetics, providing a fully informed written consent (or ascent for minors) willing to participate. The sample collection was done from midmorning to midday ( 8 hours to 12 hours) by specialised nurses from ward B02. Blood samples were transported to food and drugs control laboratory (FDCL), located at the university teaching hospitals in Lusaka, Zambia, where AAS was used to measure $\mathrm{Zn}$ levels, in the appropriate vacutainer on trays by FDCL laboratory personnel. Participants' urine samples were submitted in provided appropriate urine containers to the bacteriology laboratory, where trained microbiologists conducted urine culture.

\section{Laboratory procedures}

\section{Serum Zn determination}

About $8 \mathrm{ml}$ of blood was collected from each participant using sterile needles and syringes. Blood samples were placed into trace element heparin tubes (BD vacutainer) to be made ready for centrifugation to prepare serum. Each sample was labelled with a bar code number that corresponded to the participant's identification number. Laboratory specialists transported samples to FDCL. To prepare serum, blood samples were centrifuged for $10 \mathrm{~min}$ at $4000 \mathrm{rpm}$. The serum was then pipetted into additional tubes and stored at $-20^{\circ} \mathrm{C}$ until $\mathrm{Zn}$ determination. During $\mathrm{Zn}$ determination, refrigerated samples were thawed at room temperature and then $0.5 \mathrm{ml}$ of serum from each sample was diluted with $10 \mathrm{ml}$ of deionised water and the diluted samples were treated, each, with $1 \mathrm{ml}$ of $0.1 \%$ potassium chloride $(\mathrm{KCl})$. For the determination of $\mathrm{Zn}$ concentration, AAS technique was performed at FDCL. In this technique, five standard solutions of $\mathrm{Zn}$ were prepared in $100 \mathrm{ml}$ volumetric flasks. This was done by first preparing a stock solution by dissolving $0.1 \mathrm{~g}$ in $1000 \mathrm{ml}$ deionised water, then dilutions were made from it to make different standard solutions. The solutions were aspirated into flames and their absorption of light was measured. A standard curve of absorbance versus $\mathrm{Zn}$ concentration was plotted. Light absorbance for each sample solution was measured and the corresponding $\mathrm{Zn}$ concentration was 
determined by extrapolation from the standard curve in $\mu \mathrm{g} / \mathrm{ml}$ then converted to $\mu \mathrm{g} / \mathrm{dl}$. The data was recorded in appropriate datasheets.

\section{UTIs case definition and determination}

In our study, every participant, symptomatic or not, whose urine yielded at least 105 colony forming units (cfu) of bacterial growth from urine culture results was counted as a UTI participant.

To perform urine culture, mid-stream urine was carefully collected from all the participants and was stored in labelled sterile urine containers. ${ }^{19}$ This was done by instructing pregnant women to wash their hands and their genitals appropriately. After that, they were told to collect urine $(10-15 \mathrm{ml})$ into sterile bottles after passing the first part of the urine in the toilet. When collecting mid-stream urine, each participant was sensitised on how to collect it and its importance. The urine samples in the sterile universal bottles that were labelled were taken to the laboratory for processing within 1 hour. These samples were subjected to culture as follows: the urine sample was inoculated on air-dried plates of cysteine lactose electrolyte deficient (CLED) agar using a calibrated loop delivering $0.002 \mathrm{ml}$ of urine. Plates were incubated at $37^{\circ} \mathrm{C}$ overnight. Colony counts yielding bacterial growth of 105 $\mathrm{cfu} / \mathrm{ml}$ or more of pure isolates were deemed significant. Contaminated urine usually has less than $105 \mathrm{cfu} / \mathrm{ml}$ and often contained more than one bacteria species. ${ }^{19}$ Suspected bacterial species were characterised by colony morphology, gram stain followed by microscopic examination, and motility test. According to clinical and laboratory standard institute guidelines, isolation was identified and linked to species level using standard methods. ${ }^{19}$

\section{Socio-demographic data}

A questionnaire was used to interview participants in order to collect information. The actual demographic information collected was on age (date from last birthday), marital status (single or married), substance use (specified by the participant), an education level (as none, primary, secondary and tertiary), area of residence (as low, medium or high density) and household income (as either below 3000 or between $3000-6000$ or above 6000) at the time of data collection. Only covariates from literature findings were measured in this study.

\section{Clinical data}

A structured data collection tool was used to collect information on parity (measured as a number of times a woman gave birth to a fetus with a gestational age of at least 24 weeks) as reflecting on clinic card and history of UTIs (as either yes or no) reported by the participant. Information on gestational age was extracted from the participant's clinic card as determined by the clinician in weeks. Zn levels were measured using the AAS machine, while the UTI status was determined from urine culture results.

\section{Sample size}

Based on the prevalence of UTIs in the study setting of $16.5 \%$ we needed to enroll 212 participants in order to establish the true prevalence with $80 \%$ power and alpha level of $0.05 .{ }^{14}$ Adjusting for non-response rate of $10 \%$ the final sample size was 234 participants. Similar to other studies the sample size was sufficient to detect a difference of $10.14 \mathrm{ug} / \mathrm{dl}$ between those with and without UTIs, at an alpha level of 0.05 , assuming a mean Zn level of 108.81 $\mathrm{ug} / \mathrm{dl}$ among women with UTIs and equal standard deviation of $2.91 \mathrm{ug} / \mathrm{dl}$ in either group. ${ }^{6,20}$

\section{Statistical methods}

Data were analysed using Stata/IC version 16 (Stata Corporation, Texas, TX, USA). All continuous variables were tested for normality (using Shapiro-Wilk and qqplots). The mean with standard deviation (SD) was reported for serum $\mathrm{Zn}$ levels since data was normally distributed. Pearson Chi-square test (expected frequencies in cells was >5) or Fisher's exact test was used to test for associations between categorical variables. The student $t$ test and one way analysis of variance (ANOVA) were used to assess the mean difference of serum $\mathrm{Zn}$ between factor variables.

Furthermore, we adjusted for the effects of covariates in a multivariable logistic regression model. Firstly, we used $\mathrm{Zn}$ levels and other covariates as exposures and UTI case status as an outcome to carry out univariable logistic regression analysis. We then included factors with a $\mathrm{p}$ value below 0.20 into an unconditional logistic regression model, using investigator-led forward selection approach with a liberal $p$ value for exclusion of $p<0.15$. We assessed for effect modification and confounding using the MantelHaenszel odds ratios. We used the Hosmer Lemeshow test to assess for model goodness of fit. The significance level for all tests was set at $5 \%$ with $95 \%$ confidence intervals.

\section{RESULTS}

\section{Clinical and socio-demographic characteristics}

We had a total of 228 participants, of which $118(51.75 \%)$ had positive urine culture for UTIs, 214 (93.86\%) were married, $10(4.39 \%)$ reported using alcohol, $118(51.75 \%)$ attained tertiary education, $143(62.72 \%)$ were from a medium densely populated area, $135(59.21 \%)$ were aged 23 to 26 years, $88(38.59 \%)$ earned between 300 to 5000 ZMW, $147(64.47 \%)$ were low multiparity and 218 $(95.61 \%)$ were less than 37 weeks gestation. There was no evidence of a difference in mean serum zinc levels between women diagnosed with UTIs compared to those without UTIs (53.95 ug/dl versus $50.02 \mathrm{ug} / \mathrm{dl}, \mathrm{p}=0.407)$. Table 1 shows the study participants' clinical and socio- 
demographic characteristics according to the serum $\mathrm{Zn}$ levels.

Table 2 shows the results of the univariable analysis of factors associated with UTIs. Of the 118 women with UTI, $57(48.31 \%)$ had low serum zinc levels (below $50 \mathrm{ug} / \mathrm{dl}$ ). The majority, $72(60.50 \%)$ were from the age group 26 to
35 years, $113(94.96 \%)$ were below 37 weeks of gestation, $79(66.39 \%)$ were of low multiparity, $45(54.22 \%)$ were earning below ZMW 6000 at the time of this study, 111 $(93.28 \%)$ were married, $59(49.58 \%)$ attained a tertiary level of education, 66 (55.46) had a history of UTIs and 80 $(67.23 \%)$ were from a medium densely populated area. None of the factors reached any statistical significance from the univariate tests of association.

Table 1: Socio-demographic and clinical characteristics of participants by mean serum Zn levels.

\begin{tabular}{|c|c|c|c|c|}
\hline Factors & Level & $\mathbf{N}(\%)$ & $\begin{array}{l}\text { Mean serum Zn } \\
\text { (SD) }\end{array}$ & P value \\
\hline \multirow{2}{*}{ UTI } & No & $110(48.25)$ & $50.05(35.10)$ & \multirow{2}{*}{$0.407^{\mathrm{a}}$} \\
\hline & Yes & $118(51.75)$ & $53.92(35.10)$ & \\
\hline \multirow{2}{*}{ Marital status } & Married & $214(93.86)$ & $52.38(34.91)$ & \multirow{2}{*}{$0.607^{\mathrm{a}}$} \\
\hline & Single & $14(6.14)$ & $47.38(38.67)$ & \\
\hline \multirow{2}{*}{ Substance use } & Yes & $10(4.39)$ & $48.97(39.01)$ & \multirow{2}{*}{$0.776^{\mathrm{a}}$} \\
\hline & No & $218(95.61)$ & $52.21(34.98)$ & \\
\hline \multirow{4}{*}{ Education level } & None & $3(1.32)$ & $23.53(29.43)$ & \multirow{4}{*}{$0.240^{\mathrm{b}}$} \\
\hline & Primary & $15(6.58)$ & $53.08(38.95)$ & \\
\hline & Secondary & $92(40.35)$ & $56.47(34.61)$ & \\
\hline & Tertiary & $118(51.75)$ & $49.24(34.86)$ & \\
\hline \multirow{3}{*}{ Area density } & High & $53(23.25)$ & $53.08(34.51)$ & \multirow{3}{*}{$0.701^{b}$} \\
\hline & Low & $32(14.04)$ & $47.22(38.03)$ & \\
\hline & Medium & $143(62.72)$ & $52.78(34.77)$ & \\
\hline \multirow{3}{*}{ Age (in years) } & $16-25$ & $52(22.81)$ & $56.28(38.26)$ & \multirow{3}{*}{$0.132^{b}$} \\
\hline & $26-35$ & $135(59.21)$ & $48.27(32.30)$ & \\
\hline & $36-45$ & $41(17.98)$ & $59.24(38.70)$ & \\
\hline \multirow{2}{*}{ GA (in weeks) } & $<37$ & $218(95.61)$ & $52.02(35.00)$ & \multirow{2}{*}{$0.920^{\mathrm{a}}$} \\
\hline & $\geq 37$ & $10(4.39)$ & $53.17(38.81)$ & \\
\hline \multirow{3}{*}{ Parity } & 0 (nulliparity) & $60(26.32)$ & $50.64(34.63)$ & \multirow{3}{*}{$0.784^{\mathrm{b}}$} \\
\hline & 1-3 (low multiparity) & $147(64.47)$ & $53.19(35.36)$ & \\
\hline & $\geq 4$ (grand multipara) & $21(9.21)$ & $48.29(35.64)$ & \\
\hline \multirow{4}{*}{ Income (ZMW) } & $300-5000$ & $88(55.00)$ & $55.01(36.43)$ & \multirow{4}{*}{$0.524^{\mathrm{b}}$} \\
\hline & $6000-10000$ & $61(38.13)$ & $50.70(35.09)$ & \\
\hline & $11000-20000$ & $11(6.88)$ & $43.41(34.10)$ & \\
\hline & Missing & $68(29.82)$ & $50.90(33.80)$ & \\
\hline
\end{tabular}

Key: UTI-urinary tract infection, GA-gestational age, ZMW-Zambian kwacha at the time of study, SD-standard deviation.

Table 2: Clinical and demographic characteristic of participants; according to UTI status.

\begin{tabular}{|c|c|c|c|c|}
\hline Characteristics & Level & UTI present $\mathbf{N}(\%)$ & UTI absent $\mathbf{N}(\%)$ & P value \\
\hline \multirow{2}{*}{$\begin{array}{l}\text { Zinc deficiency }(<50 \\
\text { ug/dl) }\end{array}$} & No & $62(52.10)$ & $45(41.28)$ & \multirow{2}{*}{$0.102^{\mathrm{a}}$} \\
\hline & Yes & $57(47.90)$ & $64(58.72)$ & \\
\hline \multirow{3}{*}{ Age (in years) } & $16-25$ & $27(22.69)$ & $25(22.94)$ & \multirow{3}{*}{$0.877^{\mathrm{a}}$} \\
\hline & $26-35$ & $72(60.50)$ & $63(57.80)$ & \\
\hline & $36-45$ & $20(16.81)$ & $21(19.27)$ & \\
\hline \multirow{2}{*}{ GA (in weeks) } & $<37$ & $113(94.96)$ & $105(96.33)$ & \multirow{2}{*}{$0.613^{\mathrm{a}}$} \\
\hline & $\geq 37$ & $6(5.04)$ & $4(3.67)$ & \\
\hline \multirow{3}{*}{ Parity } & 0 (nulliparity) & $30(25.21)$ & $30(27.52)$ & \multirow{3}{*}{$0.805^{\mathrm{a}}$} \\
\hline & 1-3 (low multiparity) & $79(66.39)$ & $68(62.39)$ & \\
\hline & $\geq 4$ (grand multipara) & $10(8.40)$ & $11(10.09)$ & \\
\hline \multirow{4}{*}{ Income (ZMW) } & $300-5000$ & $45(54.22)$ & $43(55.84)$ & \multirow{4}{*}{$0.180^{\mathrm{a}}$} \\
\hline & $6000-10000$ & $35(42.17)$ & $26(33.77)$ & \\
\hline & $11000-20000$ & $3(3.61)$ & $8(10.39)$ & \\
\hline & Missing & $36(30.25)$ & $32(29.36)$ & \\
\hline
\end{tabular}




\begin{tabular}{|c|c|c|c|c|}
\hline Characteristics & Level & UTI present N (\%) & UTI absent $\mathbf{N}(\%)$ & P value \\
\hline \multirow{2}{*}{ Marital status } & Married & $111(93.28)$ & $103(94.50)$ & \multirow{2}{*}{$0.702^{\mathrm{a}}$} \\
\hline & Single & $8(6.72)$ & $6(5.50)$ & \\
\hline \multirow{4}{*}{ Education } & None & $2(1.68)$ & $1(0.92)$ & \multirow{4}{*}{$0.909^{\mathrm{b}}$} \\
\hline & Primary & $8(6.72)$ & $7(6.42)$ & \\
\hline & Secondary & $50(42.02)$ & $42(38.53)$ & \\
\hline & Tertiary & $59(49.58)$ & $59(54.13)$ & \\
\hline \multirow{2}{*}{ History of UTI } & Yes & $66(55.46)$ & $52(47.71)$ & \multirow{2}{*}{$0.242^{\mathrm{a}}$} \\
\hline & No & $53(44.54)$ & $57(52.29)$ & \\
\hline \multirow{3}{*}{ Area density } & High & $26(21.85)$ & $27(24.77)$ & \multirow{3}{*}{$0.255^{\mathrm{a}}$} \\
\hline & Medium & $80(67.23)$ & $63(57.80)$ & \\
\hline & Low & $13(10.92)$ & $19(17.43)$ & \\
\hline Overall & & $118 / 228(51.8 \%)$ & $110 / 228(48.2 \%)$ & \\
\hline
\end{tabular}

Key: UTI-urinary tract infection, a-Pearson Chi-square test, b-Fisher's exact test, GA-gestational age, ZMW-Zambian kwacha.

Table 3: Factors associated with UTI from a logistic regression model.

\begin{tabular}{|c|c|c|c|c|}
\hline Characteristics & Crude OR, 95\% CI & $P$ value & $\begin{array}{l}\text { Adjusted OR, } \\
\text { 95\% CI }\end{array}$ & $P$ value \\
\hline Zn conc (ug/dl) & $1.03(0.62-1.74)$ & 0.906 & $1.00(0.99-1.01)$ & 0.435 \\
\hline \multicolumn{5}{|l|}{ Marital status } \\
\hline Married & & & Ref & \multirow{2}{*}{0.659} \\
\hline Single & $0.79(0.27-2.36)$ & 0.690 & $1.31(0.39-4.42)$ & \\
\hline \multicolumn{5}{|l|}{ Substance use } \\
\hline Yes & & & Ref & \multirow{2}{*}{0.133} \\
\hline No & $0.45(0.11-1.76)$ & 0.250 & $0.33(0.08-1.40)$ & \\
\hline \multicolumn{5}{|l|}{ Education level } \\
\hline None & & & Ref & \\
\hline Primary & $0.57(0.04-7.73)$ & 0.687 & $0.56(0.04-8.48)$ & 0.677 \\
\hline Secondary & $1.04(0.35-3.11)$ & 0.947 & $0.52(0.40-6.31)$ & 0.607 \\
\hline Tertiary & $0.81(0.46-1.40)$ & 0.470 & $0.43(0.36-5.15)$ & 0.504 \\
\hline \multicolumn{5}{|l|}{ Area density } \\
\hline High & Ref & & Ref & \\
\hline Low & $0.71(0.29-1.73)$ & 0.459 & $0.71(0.26-1.93)$ & 0.501 \\
\hline Medium & $1.80(0.83-3.93)$ & 0.136 & $1.43(0.69-2.94)$ & 0.330 \\
\hline Age (in years) & $0.77(0.45-1.29)$ & 0.324 & $0.99(0.94-1.06)$ & 0.940 \\
\hline GA (in weeks) & $1.21(0.72-2.20)$ & 0.489 & $1.00(0.97-1.04)$ & 0.842 \\
\hline Parity & $1.16(0.69-1.96)$ & 0.574 & $1.01(0.81-1.27)$ & 0.927 \\
\hline
\end{tabular}

OR-odds ratio, 95\% CL-confidence interval.

\section{Factors associated with UTIs at the women and newborn hospital}

For a unit increase in serum $\mathrm{Zn}$ levels, the likelihood of UTIs increased by $0.3 \%$, although random chance finding could not be ruled out $(\mathrm{p}=0.435)$. Marital status, single (AOR: 0.79 , 95\% CL: 0.27-2.36) compared to married, substance use (AOR: 0.45, 95\% CI: 0.11-1.76), education level, primary (AOR: $0.57,95 \% \mathrm{CI}$ : 0.04-7.77) and tertiary (AOR: 0.81, 95\% CI: 0.46-1.40) compared to no education, area density, low (AOR: 0.71, 95\% CI: 0.29-1.73) compared to high and a unit increase in age (AOR: 0.77, 95\% CL: 0.45-1.29) reduced the odds of a UTI but this was not significant. On the other hand, education level, secondary (AOR: $1.04,95 \% \mathrm{CI}$ : 0.35-3.11) compared to no education, area density, medium (AOR: 1.804, 95\% CI: 0.83-3.93) compared to high, a unit increase in gestational age (AOR: $1.21,95 \%$ CI 0.72-2.20) and a unit increase in parity (AOR: $1.16,95 \% \mathrm{CI}: 0.69-1.96$ ) increased the odds of UTIs among pregnant women, but this was equally not significant. The results from a multivariable logistic regression are shown in Table 3.

\section{DISCUSSION}

This study sought to establish an association between maternal serum Zn levels and UTIs at women and newborn hospital in Lusaka, Zambia. Overall, UTIs were found in more than half of the pregnant women who participated in this study. Our results show that maternal serum $\mathrm{Zn}$ was 
not associated with the likelihood of UTIs among pregnant women.

Similar results were reported on the prevalence of UTIs across the globe. In Romania, a prevalence of $55 \%$ was reported, while another one in Nigeria reported a prevalence of $56 \% .^{23,24}$ UTIs were expected to be high in pregnant women as evidenced by some studies that argued that high levels of progesterone that occur during pregnancy caused high muscle contractions that led to stasis of urine flow, providing favourable conditions for the growth of UTI-causing agents. ${ }^{22}$ Routine screening of pregnant women could be done in order to prevent consequences of untreated UTIs such as preterm birth and infant mortality. ${ }^{7}$

Few studies had reported a lower prevalence of UTIs than the index study. For instance, a study in Pakistan reported a prevalence of $28.8 \%$, while a meta-analytic study of Iranian pregnant women reported a prevalence of UTIs five times lower than the index study. ${ }^{19,22}$ A plausible explanation could be that these two studies were conducted among Muslim women who practiced ablution, as clearly indicated by the study in Pakistan. ${ }^{19}$ Another reason could be methods used to determine the presence of UTIs and differences in ethno-demographic characteristics. Studies done in Kenya and Zambia with similar ethnodemographic and cultural practices reported similar prevalence rates of UTIs among pregnant women. ${ }^{14,22}$

Consistent with the extant literature, the index study did not find any relationship between serum Zn and UTIs among pregnant women. ${ }^{7,25}$ A meta-analysis on micronutrient status and their outcomes in women of reproductive age and pregnant women from Ethiopia, Kenya, Nigeria and South Africa reported no relationship between serum $\mathrm{Zn}$ status and UTIs. ${ }^{7}$ This meta-analysis was conducted on similar populations to the index study. We speculated that the findings could be due to similar kinds of soils and food types consumed in these places containing similar micronutrient composition. On the other hand, a study in Iran reported that increased serum $\mathrm{Zn}$ levels reduced the likelihood of UTIs. ${ }^{20}$ However, this study most likely reported different results to ours because of different factors. For example, the study population was predominantly Muslim non-pregnant women and was a case-control study. This meant that hygiene practices and diet were different from our population. The study also excluded participants that consumed $\mathrm{Zn}$ in the past year, which was not done in our population. The other plausible explanation could be in the different methods of $\mathrm{Zn}$ determination. $\mathrm{Zn}$ levels were determined by the BT 3000 auto analyser device and the Bio rex kit different from the index study that used AAS machine.

\section{Strengths and limitations}

The present study had a number of strengths. Firstly, the methods we used to determine serum $\mathrm{Zn}$ levels and the presence of UTIs were gold standards. Our sample size was also larger than most similar studies. Bias was minimised by using similar methods for assessing exposure in women with UTIs and those without UTIs.

Our study had a few limitations. We could not measure serum levels of other micronutrients such as iron that counteract the levels of serum $\mathrm{Zn}$ levels. ${ }^{23}$ We could not exclude women who were on iron supplements because it was a requirement for pregnant women to take such supplements at the setting. Hospital-based controls may had risk factors associated with exposure of interest and therefore, not all biases can be ruled out from the index study. The nutritional supplements during pregnancy were not documented and therefore could not be adjusted for. Furthermore, the study was conducted at a single institution in Lusaka, Zambia; therefore, as suggested by other studies, any attempts to generalise our findings to other settings in Zambia should be cautious. ${ }^{26}$

\section{CONCLUSION}

From the study findings, there was no significant association between serum $\mathrm{Zn}$ and UTIs. This result suggests that $\mathrm{Zn}$ may not be playing any role in UTIs. Micronutrient deficiencies are a serious public health concern and more robust studies are needed to confirm our findings.

\section{ACKNOWLEDGEMENTS}

We acknowledge the university of Zambia school of medicine for financial support by granting us the Moses Sinkala award. Moses Mukosha would like to acknowledge that some of his time was supported by the UNC-UNZAWits Partnership for HIV and women's reproductive health (grant number: D43 TW010558).

\section{Funding: No funding sources Conflict of interest: None declared \\ Ethical approval: The study was approved by the Institutional Ethics Committee}

\section{REFERENCES}

1. Lewicka I, Kocyłowski R, Grzesiak M, Gaj Z, Oszukowski P, Suliburska J. Selected trace elements concentrations in pregnancy and their possible role: literature review. Ginekol Pol. 2017;88(9):509-14.

2. Chababa L, Mukosha M, Sijumbila G, Vwalika B. Relationship between serum zinc levels and preeclampsia at the university teaching hospital, Lusaka, Zambia. Med J Zambia. 2016;43(3):139-44.

3. Kwangu M, Yassa P, Lambwe N, Mukosha M, Sijumbila G. Interplay between nitric oxide (NO) and glucose 6-phosphate dehydrogenase (G6PD) activity in primary hypertension. Int $\mathrm{J}$ Med Medic Sci. 2014;6(7):165-70.

4. Velasco SWE, Sanet M, Fernández-Vázquez J, Jové D, Glaría AVE, O'Halloran T, et al. A new role for zinc limitation in bacterial pathogenicity: modulation 
of $\alpha$-hemolysin from uropathogenic Escherichia coli. Scientif Reps. 2018;8(6535):1-11.

5. Angelescu K, Nussbaumer-Streit B, Sieben W, Scheibler F, Gartlehner G. Benefits and harms of screening for and treatment of asymptomatic bacteriuria in pregnancy: a systematic review. BMC Pregnancy Childbirth. 2016;16(1):336.

6. Amu E, Ndugba S, Olatona F. Knowledge of cervical cancer and attitude to cervical cancer screening among women in Somolu local government area, Lagos, Nigeria. J Commun Med Prim Health Care. 2019;31(1):76-85.

7. Abalos E, Cuesta C, Grosso AL, Chou D, Say L. Global and regional estimates of preeclampsia and eclampsia: a systematic review. Europ J Obstetr Gynecol Reproduct Biol. 2013;170(1):1-7.

8. Narváez-Caicedo GMC, Sandoval BA, Jara-Palacios MÁ. Zinc deficiency among lactating mothers from a peri-urban community of the ecuadorian andean region: an initial approach to the need of zinc supplementation. Nutrients. 2018;10(869):1-9.

9. Harika MFR, Samuel F, Kimiywe J, Mulugeta A, Eilander A. Micronutrient status and dietary intake of iron, vitamin A, iodine, folate and zinc in women of reproductive age and pregnant women in Ethiopia, Kenya, Nigeria and South Africa: a systematic review of data from 2005 to 2015 . Nutrients. 2017;9(1096):123.

10. Elzayat MA, Barnett-Vanes A, Dabour MFE, Cheng F. Prevalence of undiagnosed asymptomatic bacteriuria and associated risk factors during pregnancy: a cross-sectional study at two tertiary centres in Cairo, Egypt. BMJ Open. 2017;7(3):013198.

11. Onyango HA, Ngugi C, Maina J, Kiiru J. Urinary tract infection among pregnant women at Pumwani maternity hospital, Nairobi, Kenya: bacterial etiologic agents, antimicrobial susceptibility profiles and associated risk factors. Adv Microbiol. 2018;8(3):17587.

12. Yan YJL, Hang H, Yan B. The association between urinary tract infection during pregnancy and preeclampsia: a meta-analysis. Medicine. 2018;97(36):1-7.

13. Organisation WH: WHO recommendations on antenatal care for a positive pregnancy experience. In: reproductive health publications/ Maternal_perinatal_health. 2016: 1-152. Available at: https://www.ncbi.nlm.nih.gov/books/NBK409108/. Accessed on 3 May 2021.

14. Mukosha M, Nambela L, Mwila C, Chigunta M, Kalungia AC, Lubeya MK, et al. Urinary tract infections and associated factors in HIV infected pregnant women at a tertiary hospital in Lusaka, Zambia. Pan Afr Med J. 2020;37(328).

15. Alkema L, Chou D, Hogan D, Zhang S, Moller AB, Gemmill A, et al. Global, regional, and national levels and trends in maternal mortality between 1990 and 2015, with scenario-based projections to 2030: a systematic analysis by the UN maternal mortality estimation inter-agency group. Lancet. 2016;387(10017):462-74.

16. Ranjan STKSA, Matta N, Chokkakula S, Ansari KR. Prevalence of UTI among pregnant women and its complications in newborns. Indian $\mathrm{J}$ Pharm Pract. 2017;10(1):45-9.

17. Ashshi AM. Urinary tract infections in pregnant women, assessment of associated risk factors in Makkah, KSA. Biosci Biotechnol Res Asia. 2013;10(1):1-8.

18. Tazebew BGE, Tsegaye W, Melaku S. Associated risk factors of urinary tract infection among pregnant women at Felege Hiwot referral hospital, Bahir Dar, North West Ethiopia. BMC Res Notes. 2013;6(292).

19. Ayoyi GKAO, Bii C, Kariuki S. Prevalence, aetiology and antibiotic sensitivity profile of asymptomatic bacteriuria isolates from pregnant women in selected antenatal clinic from Nairobi, Kenya. Pan Afr Med J. 2017;26(41):1-12.

20. Brisson M, Kim JJ, Canfell K, Drolet M, Gingras G, Burger EA, et al. Impact of HPV vaccination and cervical screening on cervical cancer elimination: a comparative modelling analysis in 78 low-income and lower-middle-income countries. Lancet. 2020;395(10224):575-90.

21. Arbyn M, Weiderpass E, Bruni L, deSanjosé S, Saraiya M, Ferlay J, et al. Estimates of incidence and mortality of cervical cancer in 2018: a worldwide analysis. Lancet Glob Health. 2020;8(2):191-203.

22. Adams JW, Watts DH, Phelps BR: A systematic review of the effect of HIV infection and antiretroviral therapy on the risk of pre-eclampsia. International Journal of Gynecology \& Obstetrics. 2016; 133(1):1721.

23. Aizer A, Currie J. The intergenerational transmission of inequality: maternal disadvantage and health at birth. Science. 2014;344(6186):856-61.

24. Anggrahini SM, Notobroto HB, Suryawan A. Structural model of maternal behavior in community setting to prevent low birth weight in East Nusa Tenggara, Indonesia. Folia Medica Indonesiana. 2020;56(1):60-6.

25. Tamura T, Goldenberg RL, Freeberg LE, Cliver SP, Cutter GR, Hoffman HJ. Maternal serum folate and zinc concentrations and their relationships to pregnancy outcome. Am J Clinic Nutrit. 1992;56(2):365-70.

26. Mukosha M, Jacobs C, Musonda P, Zulu JM, Masaku $\mathrm{S}, \mathrm{Nkwemu} \mathrm{C}$, et al. Determinants of preterm births at a national hospital in Zambia: application of partial proportional odds model. Obstetr Gynecol Res. 2021;4:117-30.

Cite this article as: Musaba J, Mukosha M, Mukololo L, Vwalika B. Maternal serum zinc levels and urinary tract infections among pregnant women at a tertiary hospital in Lusaka, Zambia: a crosssectional study. Int J Basic Clin Pharmacol 2021;10:1062-8. 